Case Report

\title{
Arthroscopic Matti-russe procedure
}

\section{Abstract}

Open surgical treatment with a bone graft is considered the gold standard for the treatment of scaphoid pseudarthrosis. Arthroscopic bone fixation with bone graft placement is a minimally invasive method, which is effective and with few associated complications. To describe an arthroscopic technique for the treatment of scaphoid pseudarthrosis. The arthroscopic treatment of stable scaphoid pseudarthrosis is an effective alternative to conventional open treatment.

Keywords: Scaphoid bone; Pseudarthrosis; Wrist arthroscopy
Volume 9 Issue 2 - 2017

Tiago Pinheiro Torres, Miguel Frias, Renato Ramos, Luis Miragaia, Pedro Canela, Rui

Lemos

Centro Hospitalar de Vila Nova de Gaia, Portugal

Correspondence: Tiago Pinheiro Torres, Centro Hospitalar de Vila Nova de Gaia,Vila Nova de Gaia, Portugal, Email tiagocpt@gmail.com

\section{Introduction}

Scaphoid fractures are the most common carpal fractures with some series that indicate non-consolidation rates of up to $10 \%$. Avascular necrosis occurs in about $3 \%$ of cases, predominantly in the proximal pole. There are a number of techniques described with different types of grafts (vascularized and non-vascularized).

Matti1 in 1936 developed the technique in which by dorsal approach it crossed the proximal and distal fragments of the scaphoid and filled with spongy bone graft. Russe 2 modified the Matti technique when using the via volar to preserve the vascularization of the scaphoid and to fill with sponge graft in a single block.

The purpose of this technical note is to describe the modified MattiRusse procedure performed by arthroscopic route and percutaneous volar screw fixation.

\section{Technical note}

Case report on a 21-year-old patient with scaphoid neck pseudarthrosis with three years of evolution and with an MRI that does not reveal avascular necrosis of the proximal pole (Figures $1 \&$ 2).

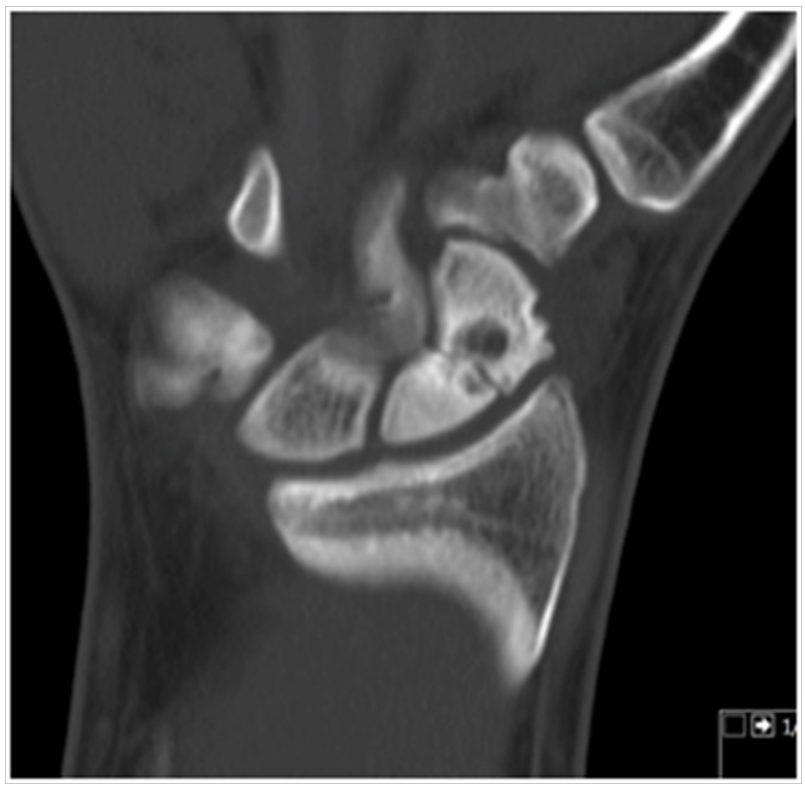

Figure I CT image showing pseudarthrosis of scaphoid waist.

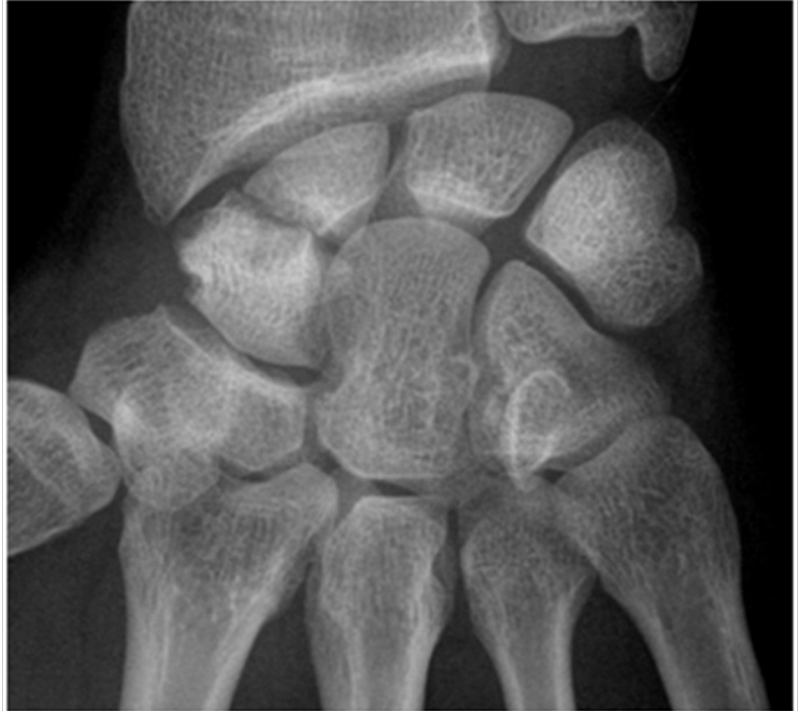

Figure $2 \times R$ image showing scaphoid non-union.

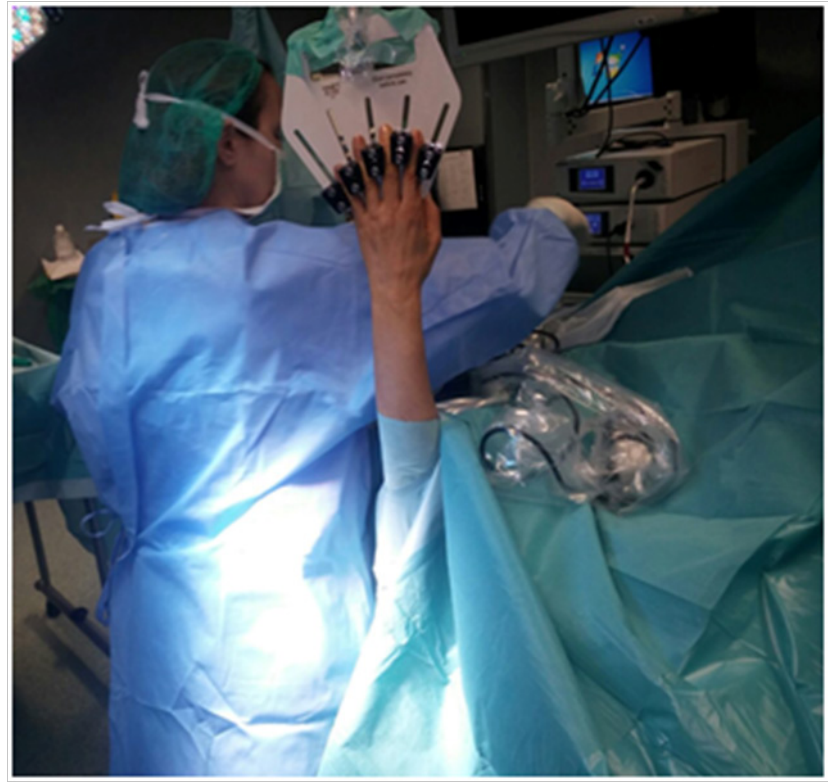

Figure 3 Placement of the patient upper limb with wrist arthroscopic materials. 
The patient was placed in the supine position with a club at the root of the arm. The anesthestic technique was loco-regional block. Than, the arthroscopic arthroscopy system was assembled with $6 \mathrm{Kg}$ traction (Figure 3).

The arthroscopic procedure started with the realization of standard radio-carpic initial portals; medium-carpal portals helped with the identification of the pseudarthrosis focus (Figure 4). The pseudarthrosis focus was blown out (Figures $5 \& 6$ ). The spongious graft of the contralateral iliac crest was harvested. Placement of the graft through the midcarpal portal of visualization by adapting the arthroscopy trocar (Figures 7-9). The traction was withdrawn. The graft was fixated with a percutaneous anterior approach and arthroscopic support and placement of the double compression screw with radiographic support (Figure 10).

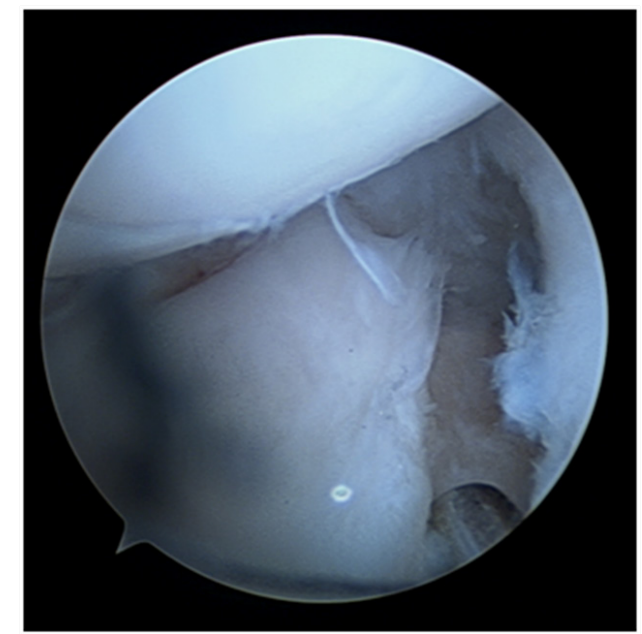

Figure 4 Arthroscopic view of pseudarthrosis.

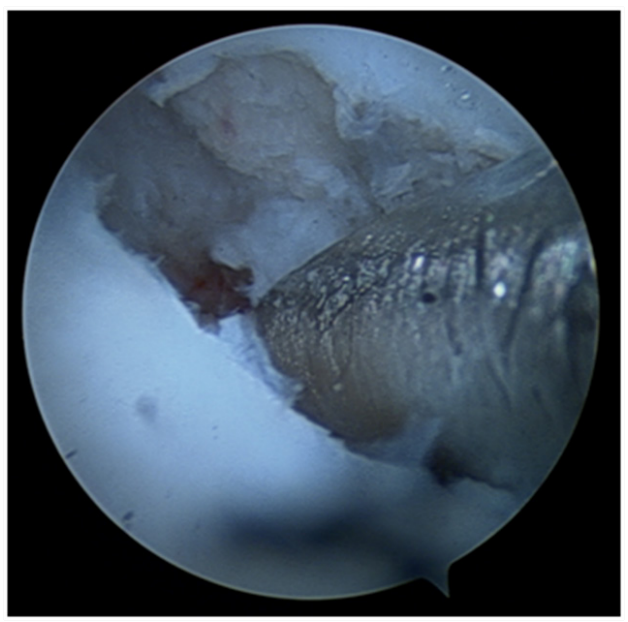

Figure 5 Arthroscopic debridement.

\section{Discussion}

Pseudarthrosis is the most common complication of scaphoid fractures. The present technique, although difficult, brings with it all the advantages inherent to arthroscopy, with smaller incisions, greater respect for vascular and ligament structures, faster recovery, less morbidity, less stiffness, direct observation of the screw placement and reduction of the focus of Pseudarthrosis. It is also associated with better functional results. This method is limited to the initial stage of pseudoarthrosis or stable fibrous union with minimal sclerosis. ${ }^{1,2}$

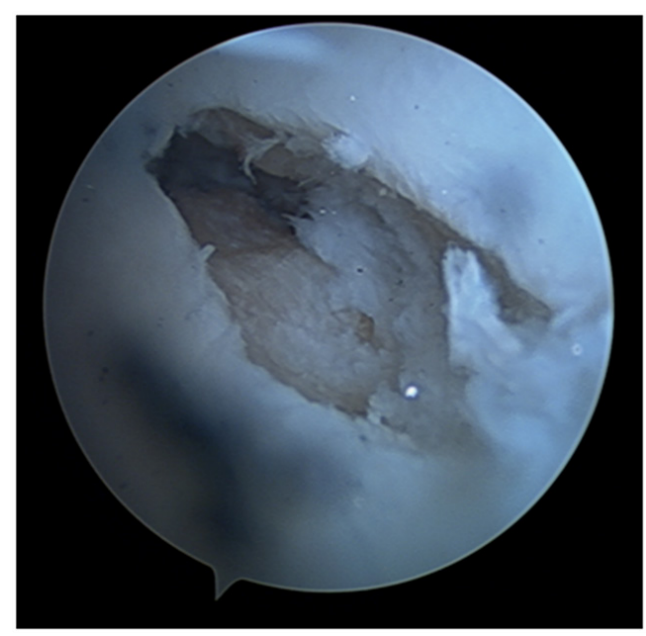

Figure 6 Arthroscopic view after debridement.

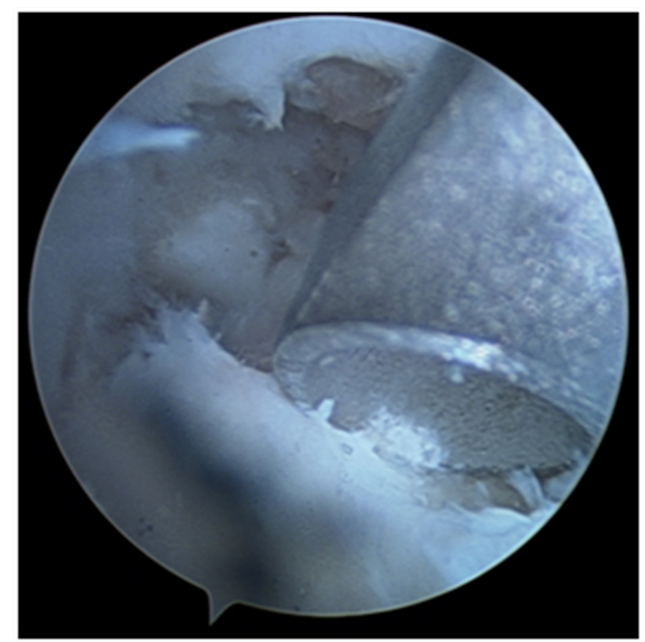

Figure 7 Arthroscopic placement of iliac crest graft with the help of the visualization trocar.

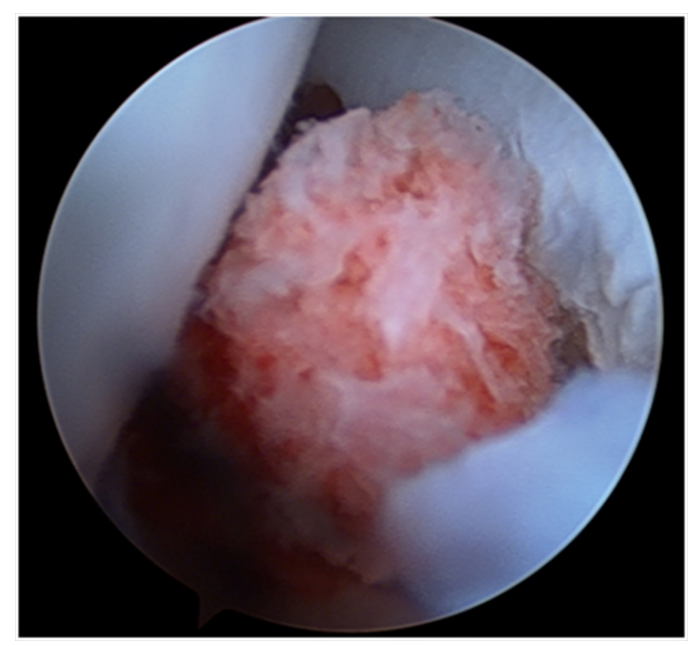

Figure 8 Bone Graft between the scaphoid pseudarthrosis.

\section{Final comments}

Although the preliminary results are satisfactory, further studies are necessary to prove the good results of this technique. 


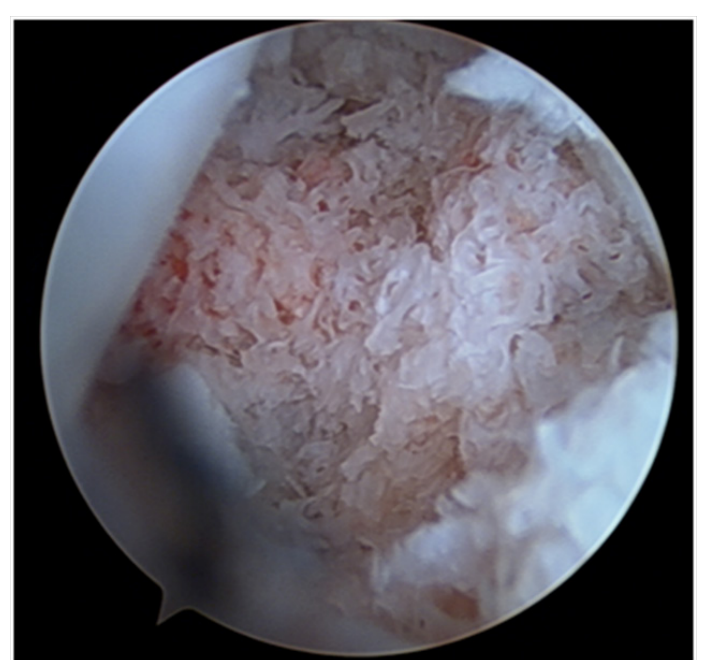

Figure 9 Arthroscopic view of the graft.

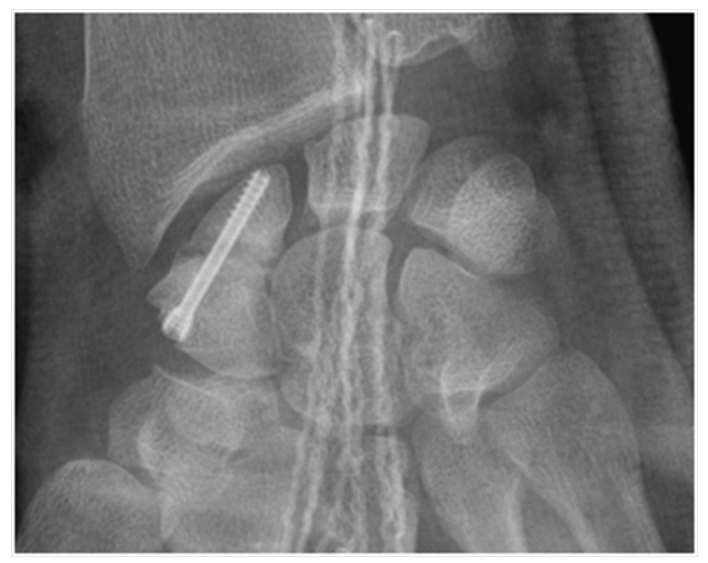

Figure 10 Post-operative $\mathrm{X}$-rays.

\section{Conflicts of interest}

The authors declare no conflict of interest.

\section{Acknowledgements}

None.

\section{References}

1. Matti H. Technik und resultatate meiner pseudarthrosen-operation. $Z$ Chir. 1936;63:1442-53.

2. Russe O. Fracture of the navicular carpal. Diagnosis, non-operative treatment, and operative treatment. $J$ Bone Joint Surg Am. 1960;42A:759-768. 